\title{
REVIEW \\ Bladder cancer in individuals with spinal cord injuries
}

\author{
B Welk ${ }^{1,2}$, A McIntyre ${ }^{3}$, R Teasell ${ }^{3,4,5}, \mathrm{P}$ Potter $^{4,5}$ and E Loh ${ }^{4,5}$
}

Study design: Prospective scoping review.

Objectives: To conduct a scoping review of all the literature related to bladder cancer in individuals with spinal cord injuries (SCI). Methods: Literature search of the databases Pubmed, CINAHL, ProQuest, PsychINFO and Scopus up to and including August 2012. Articles related to bladder cancer among SCl patients were identified, and data pertaining to epidemiology, risk factors, screening, prevention and management was reviewed and summarized.

Results: An association between bladder cancer and SCl was first reported in the 1960s, with some case reports suggesting an alarmingly high rate among SCl patients. More recent epidemiological studies have reported this risk to be substantially lower. However, bladder cancer in SCl patients tends to present at an earlier age and at a more advanced pathological stage than bladder cancer in the general population. Presenting symptoms may be atypical, and early recognition is important to improve prognosis with surgical resection. Several risk factors have been identified, including indwelling catheters, urinary tract infections and bladder calculi. Screening of SCl patients for bladder cancer is routinely recommended in many $\mathrm{SCl}$ management guidelines and by expert consensus; however, evidence for screening tools and protocols is lacking.

Conclusion: Bladder cancer is a rare, and potentially lethal occurrence in $\mathrm{SCl}$ patients. Physicians need to have a high index of suspicion for bladder cancer, particularly among SCl patients managed with long-term indwelling catheters.

Spinal Cord (2013) 51, 516-521; doi:10.1038/sc.2013.33; published online 23 April 2013

Keywords: spinal cord injury; bladder cancer

\section{INTRODUCTION}

Bladder cancer is a relatively rare disorder among the general population, with an age standardized incidence of $16 / 100000$ population, and a simple incidence of $0.02 \% .{ }^{1}$ Identified risk factors for bladder cancer in the general population include male gender, increased age, smoking, occupational exposure to certain chemicals, pelvic radiation, cyclophosphamide exposure, chronic irritation or infection, parasitic schistosomiasis infection (in endemic areas) and analgesic abuse; $90 \%$ of bladder cancer is transitional cell carcinoma (TCC), the remaining 10\% consists of squamous cell carcinoma (SCC), adenocarcinoma and other rare subtypes. $^{2}$ Bladder cancer commonly presents with hematuria and urinary symptoms, and is diagnosed with cystoscopy.

In the spinal cord-injured (SCI) population, where bladder symptoms are a significant secondary complication, the risk of bladder cancer is thought to be increased. Melzak, ${ }^{3}$ in the 1960 s, was the first to report an increased incidence of bladder cancer in a large series of SCI patients. Other studies suggested that bladder cancer contributed to $0.3-3 \%$ of the all-cause mortality of SCI patients. ${ }^{4}$ The current state of evidence surrounding bladder cancer epidemiology, risk factors, presentation, screening and treatment among SCI patients has not been reviewed. The objective of this study was to conduct a scoping review and summarize the current state of knowledge, and suggest areas for future research.

\section{MATERIALS AND METHODS}

A computer-assisted search of multiple databases (Pubmed, CINAHL, ProQuest, Scopus and PsychINFO) was conducted for experimental and clinical literature up to and including August 2012. Our search strategy was based on the preselected keywords or MeSH headings (spinal cord injury, neurogenic bladder, bladder cancer, TCC, urothelial carcinoma, squamous cell carcinoma). The inclusion criteria was any article primarily reporting on an aspect of bladder cancer among SCI patients (based on a review of the article title and abstract, and where necessary full text). This primarily included articles related to the epidemiology, risk factors, screening, treatment and outcomes of bladder cancer in SCI patients. Our only exclusion criteria was non-English language publications; given the limited literature base, no studies were excluded on the basis of publication date or research methodology. The indexing of identified articles within their identified database was reviewed to ensure other relevant indexing terms had not been missed. Individual articles selected for review were scanned for additional citations not identified by the original search. The database search results were reviewed individually by one of two authors (BW, AM), and therefore this was not a systematic literature review. A total of 21 articles were identified and included as the evidence base for this review.

\section{RESULTS}

Bladder cancer incidence among SCI patients

Multiple case series have been published examining the relationship between bladder cancer and SCI patients; they have reported simple incidences of $0.1-10 \%$ (Table 1$).^{5-17}$ Unfortunately, the majority of

${ }^{1}$ St. Joseph's Health Care, St. Joseph's Hospital, London, ON, Canada; ${ }^{2}$ Department of Surgery, Division of Urology, Western University, London, ON, Canada; ${ }^{3}$ Lawson Health Research Institute, Parkwood Hospital, London, ON, Canada; ${ }^{4}$ St. Joseph's Health Care, Parkwood Hospital, London, ON, Canada and ${ }^{5}$ Department of Physical Medicine and Rehabilitation, Western University, London, ON, Canada

Correspondence: Dr B Welk, St. Joseph's Hospital, Room B4 667, 268 Grosvenor Street, London, ON, N6A 4V2 Canada.

E-mail: blayne.welk@sjhc.london.on.ca

Received 14 November 2012; revised 20 February 2013; accepted 21 March 2013; published online 23 April 2013 
Table 1 Review of studies reporting the incidence of bladder cancer in $\mathrm{SCl}$ patients

\begin{tabular}{|c|c|c|c|c|c|c|}
\hline Lead author & Study period & Study type & Country & $\begin{array}{c}\text { Total } \\
\text { patients }\end{array}$ & $\begin{array}{c}\text { Bladder } \\
\text { cancer cases }\end{array}$ & $\begin{array}{c}\text { Simple } \\
\text { incidence }\end{array}$ \\
\hline Kaufman et al..$^{5}$ & 1975-1976 & Prospective single centre & USA & 62 & 6 & $9.68 \%$ \\
\hline Broecker et al. ${ }^{7}$ & 1963-1978 & Retrospective single centre & USA & 1052 & 10 & $0.95 \%$ \\
\hline El-Masri et al. ${ }^{6}$ & 1940-1970 & Retrospective single centre & UK & 6744 & 25 & $0.37 \%$ \\
\hline Locke et al. ${ }^{8}$ & 1983-1984 & Prospective single centre & USA & 25 & 2 & $8.00 \%$ \\
\hline Bejany et al. ${ }^{9}$ & 1971-1986 & Retrospective single centre & USA & 300 & 7 & $2.33 \%$ \\
\hline Bickel et al. ${ }^{10}$ & 1985-1990 & Retrospective multicentre & USA & 2900 & 8 & $0.28 \%$ \\
\hline Esrig et al. ${ }^{11}$ & - & Prospective single centre & USA & 37 & 2 & $5.41 \%$ \\
\hline Vereczkey et al. ${ }^{12}$ & - & Prospective multicentre & USA (veterans) & 149 & 7 & $4.70 \%$ \\
\hline West et al. ${ }^{13}$ & 1988-1992 & Retrospective multicentre & USA (veterans) & 33565 & 130 & $0.39 \%$ \\
\hline Groah et al. ${ }^{14}$ & 1950-1997 & Retrospective single centre & USA & 3670 & 21 & $0.57 \%$ \\
\hline Pannek $^{15}$ & 1995-1999 & Retrospective multicentre & Germany, Switzerland, Austria & 43561 & 48 & $0.11 \%$ \\
\hline Subramonian et al. ${ }^{16}$ & 1940-1998 & Retrospective single centre & UK & 1324 & 4 & $0.30 \%$ \\
\hline Kalisvaart et al. ${ }^{17}$ & 1983-2007 & Retrospective single centre & USA (veterans) & 1319 & 32 & $2.43 \%$ \\
\hline
\end{tabular}

Abbreviation: SCl, spinal cord injuries.

these reports did not calculate age- or gender-adjusted incidence rates, or express their results in patient-year units in order to take into account variable periods of follow-up. The three series that reported the largest incidence (5-10\%) were likely influenced by referral patterns and specific adverse risk factors, which were present within their small cohorts (which ranged from 25 to 62 patients). In addition, these case series represent an era with a different approach to the management of neurogenic bladder patients. $5,8,11$

The largest published retrospective cohort involved 43651 SCI patients from Germany, Switzerland and Austria between 1995 and $1999 .{ }^{15}$ Incidence of bladder cancer was estimated to be $0.11 \%$ based on questionnaires sent to urological departments at SCI centers. Unfortunately, details of how the departments determined the incidence was not included, which calls into question the completeness of the data. West et al. ${ }^{13}$ reported an incidence of $0.39 \%$ in a population-based assessment of 33565 veterans with SCI. Groah et al. ${ }^{14}$ reported on a population of 3670 SCI patients from a large rehabilitation hospital from 1950 to 1997, with at least 1 year follow-up. Incidence was $0.6 \%$; age-adjusted incidence of bladder cancer ranged from 19 to 77 per 100000 person years (depending on bladder management strategy), which was 15 -fold higher than the general population. In contrast, Subramonian et al. ${ }^{16}$ calculated an age-standardized incidence rate of $31 / 100000$ person years for a group of SCI patients, which was not significantly different from the general population in the same region of the United Kingdom. The four most recent publications reported a bladder cancer incidence of $0.1-2.4 \% .^{14-17}$

The variable-reported incidence of bladder cancer in individuals with SCI is likely due to several factors: different mean ages, risk factors (indwelling foley catheters, type of catheter material, smoking), screening protocols, referral patterns, statistical methods and improved survival of SCI patients over time. Determining an accurate and modern estimation of incidence in relation to the general population is an important step in assessing the risk of bladder cancer among current SCI patients.

\section{Epidemiology and mortality of bladder cancer in SCI patients}

There are several factors associated with bladder cancer in SCI patients, which suggest SCI patients may be exposed to unique risk factors, distinct from those present in the general population. The mean age at diagnosis of bladder cancer within the general population
Table 2 Studies reporting bladder cancer characteristics among samples of $\mathrm{SCl}$ patients

\begin{tabular}{|c|c|c|c|c|c|}
\hline \multirow[t]{2}{*}{ Lead author } & \multirow{2}{*}{$\begin{array}{l}\text { Mean age at } \\
\text { bladder cancer } \\
\text { diagnosis (year) }\end{array}$} & \multicolumn{3}{|c|}{ Tumor subtype } & \multirow[t]{2}{*}{$\begin{array}{l}\text { Time since } \\
\text { SCl (year) }\end{array}$} \\
\hline & & TCC & SCC & Other ${ }^{\mathrm{a}}$ & \\
\hline Kaufman et al..$^{5}$ & 51 & $0 \%$ & $17 \%$ & $83 \%$ & - \\
\hline Broecker et al. ${ }^{7}$ & 48 & $57 \%$ & $29 \%$ & $14 \%$ & 18 \\
\hline El-Masri et al. ${ }^{6}$ & 51 & $32 \%$ & $44 \%$ & $24 \%$ & 23 \\
\hline Bejany et al. ${ }^{9}$ & - & $9 \%$ & $81 \%$ & $9 \%$ & 16 \\
\hline Bickel et al. ${ }^{10}$ & 56 & $75 \%$ & $25 \%$ & $0 \%$ & 18 \\
\hline Esrig et al. ${ }^{11}$ & - & $100 \%$ & $0 \%$ & $0 \%$ & - \\
\hline Stonehill et al. ${ }^{4}$ & 58 & $29 \%$ & $59 \%$ & $12 \%$ & 32 \\
\hline West et al. ${ }^{13}$ & 57 & $55 \%$ & $33 \%$ & $12 \%$ & 24 \\
\hline Pannek ${ }^{15}$ & 53 & $81 \%$ & $19 \%$ & $0 \%$ & 23 \\
\hline Groah et al. ${ }^{14}$ & 48 & - & - & - & 20 \\
\hline Hess et al. ${ }^{18}$ & 61 & $44 \%$ & $38 \%$ & $19 \%$ & 23 \\
\hline Subramonian et al. ${ }^{16}$ & 59 & $25 \%$ & $50 \%$ & $25 \%$ & 22 \\
\hline Kalisvaart et al. ${ }^{17}$ & - & $31 \%$ & $47 \%$ & $22 \%$ & 34 \\
\hline
\end{tabular}

Abbreviations: SCC, squamous cell carcinoma; SCI, spinal cord injuries; TCC, transitional cell carcinoma.

aOther pathological subtypes include mixed TCC/SCC, adenocarcinoma, undifferentiated.

is $60-70$ years. $^{2}$ However, the mean age of diagnosis in SCI patients is between 48 and 61 years (Table 2). ${ }^{4-7,9-11,13-18}$ A large series by Pannek ${ }^{15}$ reported a mean age of bladder cancer diagnosis of 53 years; similarly, Subramonian et al. ${ }^{16}$ found a mean age of diagnosis at 48 years.

There appears to be a consistent relationship between the time of the SCI and the diagnosis of bladder cancer. The majority of studies report a consistent mean of 18-24 years from the onset of the SCI to the bladder cancer diagnosis (Table 2). One study reported a shorter mean time of 16 years, ${ }^{9}$ and two reported slightly longer mean times of 32-34 years. ${ }^{4,17}$ The prolonged time lapse between SCI and bladder cancer may be the result of a generally young population at the time of SCI who would be unlikely to develop bladder cancer. However, the younger than expected mean age of diagnosis suggests there is an independent risk factor involved in SCI patients, which is distinct from the general population. 
The bladder tumor subtype is another indicator of a unique risk factor among SCI patients. In the general population the estimated frequency of SCC is $2-7 \% .{ }^{19}$ SCC of the bladder is commonly associated with factors that produce excessive bladder inflammation, such as parasitic infection with schistosomiasis. ${ }^{20,21}$ SCI-related series of bladder cancer have almost uniformly demonstrated an increased number of SCC tumors (25-81\%, Table 2). There also may be an increase in TCC; Pannek's ${ }^{15}$ series found that $81 \%$ of the bladder cancer histology was traditional TCC, and Esrig et al. ${ }^{11}$ found only TCC within his small series. One possible explanation of these findings is that the initial tumor is pathologically TCC, which then differentiates into a squamous subtype, which is difficult to characterize pathologically. ${ }^{13}$

Bladder cancer within SCI patients also appears to follow a different natural history. In the general population, $\sim 25 \%$ of new bladder cancers are muscle invasive at presentation. ${ }^{2}$ Bladder muscle invasion is the primary factor that determines the need for surgical removal of the entire bladder. In SCI patients with bladder cancer, $58-100 \%$ of patients present with muscle invasive disease (Table 2). This is significantly higher when compared with the general population. Whether this is due to the increased incidence of SCC (which also tends to present at higher stage in the general population ${ }^{19}$ ) or is specific to SCI patients who develop bladder cancer is unclear. The series of Kalisvaart et al. ${ }^{17}$ has the largest number of patients with bladder cancer and histological details, and $70 \%$ of patients with TCC died at a mean of 1.7 years and $73 \%$ of those with SCC died at a mean of 5 years after diagnosis.

Bladder cancer-specific mortality has been reported to range from 12 to $57 \% .^{4,10,14-18}$ In a well-controlled study by Groah et al., ${ }^{14}$ the standardized mortality ratio was 71-fold higher than that of the general population. A study by West et al. ${ }^{13}$ reported a 1-year overall survival rate after treatment of bladder cancer of $61 \%$; similar results were found after 1 year by Stonehill et al. ${ }^{4}$ (70\%). Broecker et al. ${ }^{7}$ reported that the median survival period of 10 bladder cancer patients with SCI was 13 months. Hess et al. ${ }^{18}$ reported that among 16 patients with SCI, the median survival time was only 8 months. Groah et al. ${ }^{22}$ analyzed a series of 20 patients with SCI and bladder cancer, and separated them into those that survived $>5$ years from diagnosis and those who died due to bladder cancer within 5 years. When examining risk factors (smoking, indwelling catheter use, stones, urinary infections), more of these factors tended to be present in the group that died from their disease. The increased mortality from bladder cancer among SCI patients may be partly explained by the increased clinical stage at presentation, and the comorbidities associated with this population. SCI patients may not be diagnosed as quickly as patients in the general population, owing to symptoms such as gross hematuria being attributed to catheter trauma or urinary infection.

\section{Risk factors for development of bladder cancer in SCI patients}

Much of the research on bladder cancer in SCI patients has focused on determining whether the use of an indwelling catheter results in an increased risk of bladder cancer. Historically, indwelling catheters were routinely used in SCI patients, until it was recognized that indwelling catheters were associated with a significantly higher risk of death due to infection and renal dysfunction. ${ }^{23}$ Concurrently, evidence emerged suggesting that indwelling catheters were associated with an increased risk of bladder cancer. ${ }^{4}$ This lead to the increased use of intermittent catheterization or external collection devices to manage the bladders of SCI patients. ${ }^{24}$ Animal models suggest that catheters have a proneoplastic effect on the bladder urothelium; chronic irritation, such as that because of an indwelling catheter, causes epithelial hyperplasia, which leads to an increased risk of malignant clonal cells emerging. ${ }^{25-27}$ This is also hypothesized to be the pathogenesis of bladder cancer associated with schistosomiasis infection. ${ }^{21}$

The prevailing belief is that indwelling catheters, particularly when used for $>10$ years, are a risk factor for bladder cancer. ${ }^{28-30}$ Most series of SCI patients with bladder cancer show that a high proportion used indwelling catheters (50-100\%). 4,7,9,10,13,16-18 Data from the US National Spinal Cord Injury Database suggest that although intermittent catheterization has increased in popularity since the 1970s, few patients continue to use it over long-term follow-up, whereas patients who use an indwelling catheter are likely to continue to use this strategy long term. ${ }^{31}$ Groah et al. ${ }^{14}$ demonstrated an increased age-standardized rate of bladder cancer in patients with primarily indwelling catheters usage for $>1$ year; the relative risk of bladder cancer was 4.6-fold higher in patients with catheters for 10-19 years versus those with catheters for 1-9 years. However, among the 37 SCI patients with bladder cancer in the series by Pannek, ${ }^{15}$ only $19 \%$ currently managed their bladder with an indwelling catheter (an additional 14\% had a history of previous catheter usage). West et al. ${ }^{13}$ reported that in the group of SCI bladder cancer patients, those without catheters accounted for 38\% of the bladder tumors, and these patients tended to develop bladder cancer more rapidly than their SCI counterparts with indwelling catheters. These conflicting reports are probably compounded by the retrospective nature of the studies, and the fact that bladder management tends to change over a patient's lifetime. Vereczkey et al. ${ }^{12}$ attempted to analyze 31 potential risk factors and interactions for bladder cancer in a group of 153 SCI patients; unfortunately given the low event rate, this model was over-fitted to the data, and its results are likely unreliable.

The second risk factor associated with bladder cancer among SCI patients is that of chronic urinary tract infections (UTI). Overall, UTIs are more common in SCI patients, occurring on average 2.5 times per patient per year. ${ }^{32}$ Animal models have demonstrated that there is an increased production of nitrosamine during infection with bacterial strains of Escherichia coli and Proteus; this causes urothelial hyperplasia and neoplasia. ${ }^{33}$ Retrospective studies have demonstrated an almost uniform increased risk of bladder cancer associated with frequent $\mathrm{UTI}^{20}$ It is not surprising that this association is also seen in the SCI population. Two studies reported uniform bacteriuria among patients that developed bladder cancer, ${ }^{5,9}$ and Pannek ${ }^{15}$ reported $41 \%$ of patients with bladder cancer had bacteriuria and a $24 \%$ had a history of frequent urinary infections. Unfortunately, it is extremely difficult to accurately quantify the number of urinary infections in this population, owing to the high prevalence of asymptomatic bacteriuria, and the detection and recall bias that is associated with retrospective studies.

Three series of SCI patients with bladder cancer reported bladder stones in $33-59 \%$ of patients (another potential cause of bladder inflammation) ${ }^{4,5,9}$ It is not clear whether this is an independent risk factor, or an association with indwelling catheters or UTIs. Groah et al. ${ }^{14}$ addressed this with a multivariable regression model and did not demonstrate that bladder calculi are a significant independent risk factor for bladder cancer.

Smoking is the major risk factor for bladder cancer in the general population. Smoking rates in SCI patient series vary widely. Some SCI populations are predominately veterans, who have a higher rate of smoking than the general population..$^{13}$ However, Hess et al. ${ }^{18}$ did not report a difference in smoking rates between their sample of SCI veterans and SCI non-veterans. Four additional studies found that the 
proportion of SCI patients who were smokers ranged from 35 to $79 \%{ }^{4,14-18}$

Other theories as to why SCI patients may be more prone to developing bladder cancer include an increased urine contact time, due to less frequent voiding, altered growth factor expression, and hormonal and immune system changes (see Table 3 for a summary of risk factors). ${ }^{34}$ It is also possible that denervation of the bladder leads to the development of urothelial dysplasia.

\section{Bladder cancer diagnosis and screening}

When examining bladder cancer among SCI patients, it is important to critically examine the tools physicians use (such as urinalysis, urine cytology, cystoscopy and random bladder biopsies) to detect bladder cancer in this population, and to determine whether there is a role for screening with any of these investigations.

The majority of patients in the published series presented with traditional symptoms, such as gross hematuria, bladder mass, or hydronephrosis/renal failure (Table 4). ${ }^{4-6,8-11,14,16-18,29}$ It is important to note that SCI patients with bladder cancer can present with less specific signs or symptoms, such as frequent UTIs, bladder calculi, penile discharge or scrotal infection. These symptoms, although commonly due to benign causes, should lead to a thorough evaluation for bladder cancer. Only a few case series include patients that were diagnosed with bladder cancer as a result of screening. $4,5,11,14,17$ The largest absolute number of patients identified by a screening protocol was $8 / 32$ bladder cancer cases, although

Table 3 Potential risk factors for bladder cancer among $\mathrm{SCl}$ patients

Indwelling catheter usage (time-dependent risk factor)

Chronic UTIs

Bladder calculi

Smoking

Increased urine contact time

Altered immunological function

Abbreviations: SCl, spinal cord injuries; UTI, urinary tract infection.

Table 4 Presenting symptoms associated with bladder cancer presentation among $\mathrm{SCl}$ patients

\begin{tabular}{|c|c|c|c|c|c|}
\hline \multirow[t]{2}{*}{ Lead author } & \multirow{2}{*}{$\begin{array}{l}\text { Patients with } \\
\text { bladder cancer }\end{array}$} & \multicolumn{4}{|c|}{ Symptom leading to diagnosis } \\
\hline & & Traditionala & $\begin{array}{c}\text { Frequent } \\
\text { UTIs }\end{array}$ & Stones & $\begin{array}{c}\text { Scrotal } \\
\text { abscess/ } \\
\text { purulent } \\
\text { penile } \\
\text { drainage }\end{array}$ \\
\hline
\end{tabular}

\begin{tabular}{|c|c|c|c|c|c|}
\hline Kaufman et al. ${ }^{5}$ & 6 & $83 \%$ & & & \\
\hline El-Masri et al. ${ }^{6}$ & 25 & $72 \%$ & $20 \%$ & & \\
\hline Locke et al. ${ }^{8}$ & 2 & $100 \%$ & & & \\
\hline Bejany et al. ${ }^{9}$ & 11 & $72 \%$ & & $45 \%$ & \\
\hline Bickel et al. ${ }^{10}$ & 8 & $87 \%$ & $13 \%$ & & \\
\hline $\begin{array}{l}\text { Esrig et al. } \\
\text { Stonehill et al. }\end{array}$ & $\begin{array}{r}2 \\
17\end{array}$ & $65 \%$ & & $18 \%$ & $6 \%$ \\
\hline Navon et al. ${ }^{29}$ & 14 & $69 \%$ & $31 \%$ & & \\
\hline Groah et al. ${ }^{14}$ & 20 & $63 \%$ & & & \\
\hline Hess et al. ${ }^{18}$ & 16 & $100 \%$ & & & \\
\hline Subramonian et al. ${ }^{16}$ & 4 & $75 \%$ & $25 \%$ & & \\
\hline Kalisvaart et al. ${ }^{17}$ & 32 & $37 \%$ & & & $11 \%$ \\
\hline
\end{tabular}

Not all rows add to $100 \%$ because of some patients being asymptomatic or the presenting symptoms not being reported for all patients.

aTraditional symptoms include hematuria, suprapubic mass, renal failure or hydronephrosis it is not clear whether patients also reported symptoms at the time of screening cystoscopy. ${ }^{17}$

Navon et al. ${ }^{29}$ reviewed 14 SCI patients with SCC of the bladder diagnosed between 1980 and 1996. Five cases were asymptomatic at diagnosis, and found because of surveillance cystoscopy (although these were not truly screening procedures as these cystoscopies were performed because of frequent UTIs). There was a trend toward higher stage disease and mortality in those patients that presented with symptoms (for example, mass, hematuria and renal failure). A urological screening program was carried out in 500 SCI patients; cystoscopy was carried out in those who used some form of urinary catheter for at least 5 or 10 years (depending on smoking status). ${ }^{35}$ No cancers were detected with the screening protocol. Three patients from this cohort were diagnosed with bladder cancer outside of the screening protocol; two of these patients did not meet the criteria for yearly cystoscopy, as they used external urine collection devices, and the remaining patient had a normal cystoscopy and biopsy 4 months before their diagnosis as part of the screening protocol. This series also demonstrated that the real-world effectiveness of yearly screening cystoscopy is poor; patients only completed an average of 2.6 cystoscopies over 6 years (as opposed to the expected 6 cystoscopys over 6 years).

Noninvasive screening methods have been investigated in SCI patients. Urinalysis has been studied as a screening tool among older males in the general population; ${ }^{36}$ however, its usefulness in the SCI population is limited because of the difficulty in interpreting the results in SCI patients (because of chronic bacteriuria and hematuria from catheter use). Stonehill et al. ${ }^{30}$ sought to determine the effectiveness of urine cytology at detecting bladder cancer in SCI patients. Two hundred and eight SCI patients (primarily managed with indwelling catheters) had urine cytologies analyzed, and then bladder biopsies performed. Cytology demonstrated a specificity of $71 \%$ and sensitivity of $91 \%$ for detecting bladder cancer. Other series have identified bladder cancers with positive cytology. ${ }^{3,8}$ This is refuted by several authors who found cytology to have a very low diagnostic yield. ${ }^{5-7,9,10}$ Davies et al. ${ }^{37}$ analyzed annual urine samples from a cohort of 457 SCI patients (3 patients were diagnosed with superficial TCC) and found that cytology, bladder tumor-associated antigen and survivin urinary markers did not detect any of these cases. The usefulness of cytology likely depends on the ability of the cytopathologist to interpret samples with background inflammation. ${ }^{30}$

In the general population, screening cystoscopies are the standard of care after an initial diagnosis of noninvasive bladder cancer. Many series have advocated the use of cystoscopy as an annual screening tool in specific groups of SCI patients $7,9,11,13,17,38$ others have suggested yearly cytology. ${ }^{8}$ However, some studies have shown that screening did not lead to an earlier diagnosis for patients that were later diagnosed with bladder cancer. ${ }^{22,30}$ SCI patients who survived their diagnosis of bladder cancer actually had less screening cystoscopies performed than those who died from the disease. ${ }^{22}$ In order to enhance the yield of cystoscopy, some authors have advocated random bladder biopsies at the time of cystoscopy to identify occult pathology and to assess for future bladder cancer risk. ${ }^{4,5,9,11,38}$

For a screening program to be effective, the disease must have substantial morbidity or mortality, have a high prevalence, be detectable by an acceptable method and have the possibility of improved treatment because of early detection. The evidence suggests that there is indeed a substantial mortality associated with the diagnosis of bladder cancer in SCI patients. However, the prevalence 
Table 5 Summary points

Summary points

1 Recently, large series of bladder cancer diagnosis among SCl patients estimate an incidence of $0.1-2.4 \%$, or an age-standardized incidence rate of $19-77 / 100000$ person-years.

2 Bladder cancer in SCl patients tends to present at an earlier age and a more advanced stage than that in the general population. Patients are more likely to develop SCC-type bladder cancer, on average 18-24 years after the onset of SCI.

3 Associated risk factors for bladder cancer specific to this population may include indwelling catheters, UTIs and bladder stones. Smoking history is an important risk factor in the general population, and among SCl patients.

4 The use of routine screening urine cytology, cystoscopy and random bladder biopsy has not been shown to significantly influence mortality. These screening tests do not fulfill many of the criteria of a screening test: the incidence of bladder cancer in this population is relatively small, bladder cancer is not as easy to detect using conventional tools (owing to the abnormal appearance and histological changes of the SCl bladder), and bladder cancer can occur rapidly, making yearly screening less likely to detect the disease at an earlier stage.

Abbreviations: SCC, squamous cell carcinoma; SCI, spinal cord injuries.

of bladder cancer is likely not as high as once believed, and our ability to detect bladder cancer in SCI patients at a stage where it will improve outcomes is questionable. Yearly cystoscopy provides only a single snapshot of a patient's bladder. Given the aggressive bladder tumors that can present in SCI patients, cystoscopies may not occur at a time to significantly influence management. Despite this, $95 \%$ of Japanese urologists believe in annual cystoscopy for SCI patients, ${ }^{39}$ and an American survey found that 25\% of urologists perform routine cystoscopy on all SCI patients whereas $75 \%$ perform yearly cystoscopy if the patient uses an indwelling catheter. ${ }^{40}$ To date, guidelines have not recommended specific strategies to mitigate the morbidity and mortality associated with bladder cancer. The Paralyzed Veterans Association guideline for bladder management suggests that physicians consider 'more frequent cystoscopic evaluations for individuals with chronic indwelling catheters' because of the risk of bladder cancer, ${ }^{41}$ and the Spinal Cord Injury Rehabilitation evidence project suggests that 'screening for cancer may require routine biopsy as well as cystoscopy' ${ }^{42}$ Guidelines in the urological literature do not specifically address bladder cancer in this population, ${ }^{43}$ but rather suggest that 'annual cystoscopy and biopsy do not fit the criteria for a screening test and cannot be recommended even in high risk groups. ${ }^{28}$

The molecular and genetic understanding of TCC has improved significantly over the past decade (based on study in non-SCI patients). Fluorescence in-situ hybridization assays for specific chromosomal abnormalities, PCR-based assays for molecular mutations and gene expression analysis are being studied as possible ways to aid in the detection and risk stratification of non-SCI patients at risk for bladder cancer. ${ }^{44}$ Advances in the imaging field have lead to the development of fluorescence cystoscopy, which utilizes a contrast mechanism to identify bladder pathology, and Raman spectroscopy, which indicates tissue of similar molecular composition based on the change in their vibrational energy after exposure to light. ${ }^{45}$ Whether these new developments can be applied to SCI patients (given their unique presentation and histology) remain to be seen, and provide exciting future directions for research in this area.

\section{CONCLUSIONS}

Bladder cancer is a significant oncologic complication in SCI patients. The literature suggests that bladder cancer occurs more often in SCI patients than that in the general population, and is associated with significant mortality (Table 5).

A high level of suspicion is required for SCI patients with symptoms such as hematuria, obstructive renal failure, suprapubic mass, frequent urinary infections or purulent urethral discharge, as may be due to bladder cancer. We recommend that these patients be referred to a urologist for further investigation, which may include urinalysis and cytology, cystoscopy and abdominal imaging in the form of a contrast computed tomography or ultrasound, preferably with a full bladder.

Patients with a long-standing SCI, smoking history, a history of frequent UTIs or indwelling catheter usage for $>5$ years may be at the highest risk for bladder cancer. Although screening these patients is a common practice, there is no evidence that the use of annual urinary markers or cystoscopy is effective, or that these investigations meet the principles of a screening test. However, they are generally minimally invasive tests, and the potential benefit of detecting an early malignancy may outweigh the potential risks associated with screening practices in this population. Further research is required to assess independent risk factors for bladder cancer among SCI patients, in order to identify high-risk populations for potential surveillance strategies.

\section{CONFLICT OF INTEREST}

The authors declare no conflict of interest.

1 Society CC. Canada Cancer Society Steering Committee: Canadian Cancer Statistics 2010. Toronto, 2010.

2 Silverman D, Hartge P, Morrison A, Devesa S. Epidemiology of bladder cancer. Hematol Oncol Clin North Am 1992; 6: 1-30.

3 Melzak J. The incidence of bladder cancer in paraplegia. Paraplegia 1966; 4: 85-96.

4 Stonehill W, Dmochowski R, Patterson A, Cox C. Risk factors for bladder tumors in spinal cord injury patients. J Urol 1996; 155: 1248-1250.

5 Kaufman J, Fam B, Jacobs SC, Gabilondo F, Yalla S, Kane JP et al. Bladder cancer and squamous metaplasia in spinal cord injury patients. J Urol 1977; 118: 967-971.

6 El-Masri WS, Fellows G. Bladder cancer after spinal cord injury. Paraplegia 1981; 19: 265-270.

7 Broecker B, Klein F, Hackler R. Cancer of the bladder in spinal cord injury patients. J Urol 1981; 125: 196-197.

8 Locke J, Hill D, Walzer Y. Incidence of squamous cell carcinoma in patients with long-term catheter drainage. J Urol 1985; 133: 1034-1035.

9 Bejany D, Lockhart J, Rhamy R. Malignant vesical tumors following spinal cord injury. J Urol 1987; 138: 1390-1392.

10 Bickel A, Culkin D, Wheeler JJ. Bladder cancer in spinal cord injury patients J Urol 1991; 146: 1240-1242.

11 Esrig D, Mcevoy K, Bennett C. Bladder cancer in the spinal cord-injured patient with Iong-term catheterization: a casual relationship? Semin Urol 1992; 10: 102-108.

12 Vereczkey Z, Schmeidler J, Binard J, Bauman W. Bladder cancer risk in patients with spinal cord injury. J Spinal Cord Med 1998; 21: 230-239.

13 West D, Cummings J, Longo W, Virgo K, Johnson F, Parra R. Role of chronic catheterization in the development of bladder cancer in patients with spinal cord injury. Urology 1999; 53: 292-297.

14 Groah S, Weitzenkamp D, Lammertse D, Whiteneck G, Lezotte D, Hamman R. Excess risk of bladder cancer in spinal cord injury: evidence for an association between indwelling catheter use and bladder cancer. Arch Phys Med Rehabil 2002; 83 346-351. 
15 Pannek J. Transitional cell carcinoma in patients with spinal cord injury: a high risk malignancy? Urology 2002; 59: 240-244.

16 Subramonian K, Cartwright R, Harnden P, Harrison S. Bladder cancer in patients with spinal cord injuries. BJU Int 2004; 93: 739-743.

17 Kalisvaart J, Katsumi H, Ronningen L, Hovey R. Bladder cancer in spinal cord injury patients. Spinal Cord 2010; 48: 257-261.

18 Hess M, Zhan E, Foo D, Yalla S. Bladder cancer in patients with spinal cord injury. J Spinal Cord Med 2003; 26: 335-338.

19 Manunta A, Vincendeau S, Kiriakou G, Lobel B, Guille F. Non-transitional cell bladder carcinomas. BJU Int 2005; 95: 497-502.

20 Abol-Enein H. Infection: is it a cause of bladder cancer? Scand J Urol Nephrol Suppl 2008; 218: 79-84.

21 Rosin M, Anwar W, Ward A. Inflammation, chromosomal instability, and cancer: the schistosomiasis model. Cancer Res 1994; 54: 1929s-1933s.

22 Groah S, Lammertse D. Factors associated with survival after bladder cancer in spinal cord injury. J Spinal Cord Med 2003; 26: 339-344.

23 Donnelly J, Hackler R, Bunts R. Present urologic status of the World War II paraplegic: 25-year followup. Camparison with status of the 20-year Korean War paraplegic and 5-year Vietnam paraplegic. J Urol 1972; 108: 558-562.

24 Lapides J, Diokno A, Silber S, Clean Lowe B. intermittent self-catheterization in the treatment of urinary tract disease. J Urol 1972; 107: 458-461.

25 Akaza H, Murphy W, Soloway M. Bladder cancer induced by noncarcinogenic substances. J Urol 1984; 131: 152-155.

26 Kawai K, Kawamata H, Kemeyama S, Rademaker A, Oyasu R. Persistence of carcinogen-altered cell population in rat urothelium which can be promoted to tumors by chronic inflammatory stimulus. Cancer Res 1994; 54: 2630-2632.

27 Murphy W, Blatnik A, Shelton T, Soloway M. Carcinogenesis in mammalian urothelium: changes induced by non-carcinogenic substances and chronic indwelling catheters. J Urol 1986; 135: 840-844.

28 Cameron A, Rodriguez G, Schomer K. Systematic review of urological followup after spinal cord injury. J Urol 2012; 187: 391-397.

29 Navon J, Soliman H, Khonsari F, Ahlering T. Screening cystoscopy and survival of spinal cord injured patients with squamous cell cancer of the bladder. J Urol 1997; 157: 2109-2111.

30 Stonehill W, Goldman H, Dmochowski R. The use of urine cytology for diagnosing bladder cancer in spinal cord injured patients. J Urol 1997; 157: 2112 2114.

31 Cameron A, Wallner L, Tate D, Sarma A, Rodriguez G, Clemens J. Bladder management after spinal cord injury in the United States 1972 to 2005. J Urol 2010; 184: 213-217.
32 Siroky MB. Pathogenesis of bacteriuria and infection in the spinal cord injured patient. Am J Med 2002; 113 (Suppl IA), 67S-79S.

33 Davis C, Cohen M, Hackett R, Anderson M, Warren M. Urothelial hyperplasia and neoplasia. III. Detection of nitrosamine production with different bacterial genera in chronic urinary tract infections of rats. J Urol 1991; 145: 875-880.

34 Cruse J, Lewis RJ, Bishop G, Kliesch W, Gaitan E, Britt R. Decreased immune reactivity and neuroendocrine alterations related to chronic stress in spinal cord injury and stroke patients. Pathobiology 1993; 61: 183-192.

35 Yang C, Clowers D. Screening cystoscopy in chronically catheterized spinal cord injury patients. Spinal Cord 1999; 37: 204-207.

36 Messing E, Young T, Hunt V, Roecker EB, Vaillancourt AM, Hisgen WJ et al. Home screening for hematuria: results of a multiclinic study. J Urol 1992; 148: 289-292.

37 Davies B, Chen J, Mcmurry T, Landsittel D, Lewis N, Brenes G et al. Efficacy of BTA stat, cytology, and survivin in bladder cancer surveillance over 5 years in patients with spinal cord injury. Urology 2005; 66: 908-911.

38 Delnay K, Stonehill W, Goldman H, Jukkola A, Dmochowski R. Bladder histological changes associated with chronic indwelling urinary catheter. J Urol 1999; 161: 1106-1108.

39 Kitahara S, Iwatsubo E, Yasuda K, Ushiyama T, Nakai H, Suzuki $T$ et al. Practice patterns of Japanese physicians in urologic surveillance and management of spinal cord injury patients. Spinal Cord 2006; 44: 362-368.

40 Razdan S, Leboeuf L, Meinbach D, Weinstein D, Gousse A. Current practice patterns in the urologic surveillance and management of patients with spinal cord injury. Urology 2003; 61: 893-896.

41 Linsenmeyer T, Bodner D, Creasey G, Green B, Groah S, Joseph A. Bladder management for adults with spinal cord injury: a clinical practice guideline for health-care providers. J Spinal Cord Med 2006; 29: 527-573.

42 Wolfe DL, McIntyre A, Cheung K, Goettl T, Walia S, Loh E et al. Bladder Health and Function Following Spinal Cord Injury. In Eng JJ TRW, Miller WC, Wolfe DL, Townson AF, Hsieh JTC, Connolly SJ, Mehta S and Sakakibara BM (eds). Spinal Cord Injury Rehabilitation Evidence. Version 4.0. Vancouver, 2012.

43 Abrams P, Agarwal M, Drake M, El-Masri W, Fulford S, Reid S et al. A proposed guideline for the urological management of patients with spinal cord injury. BJU Int 2008; 101: 989-994.

44 Netto G. Molecular biomarkers in urothelial carcinoma of the bladder: are we there yet? Nat Rev Urol 2012; 9: 41-51.

45 Cauberg E, De Bruin DM, Faber D, Van Leeuwen TG, De La Rosette JJ, De Reijke TM. A new generation of optical diagnostics for bladder cancer: technology, diagnostic accuracy, and future applications. Eur Urol 2009; 56: 287-296. 